\title{
Effects of glucocorticoids on human brown adipocytes
}

\author{
Johanna L Barclay 1,2,3, Hadiya Agada ${ }^{1,3}$, Christina Jang ${ }^{1,4}$, Micheal Ward ${ }^{2,3}$, \\ Neil Wetzig ${ }^{4}$ and Ken K Y Ho ${ }^{1,3,4}$ \\ ${ }^{1}$ School of Medicine, University of Queensland, Herston, Queensland, Australia \\ ${ }^{2}$ Mater Research Institute, The University of Queensland at TRI, South Brisbane, Queensland, Australia \\ ${ }^{3}$ The Translational Research Institute, 37 Kent Street, Woolloongabba, Brisbane, Queensland, Australia \\ ${ }^{4}$ Princess Alexandra Hospital, Brisbane, Queensland, Australia
}

Correspondence

should be addressed

to $\mathrm{K} \mathrm{K} \mathrm{Y} \mathrm{Ho}$

Email

k.ho@uq.edu.au

\begin{abstract}
Clinical cases of glucocorticoid (GC) excess are characterized by increased fat mass and obesity through the accumulation of white adipocytes. The effects of GCs on growth and function of brown adipose tissue are unknown and may contribute to the negative energy balance observed clinically. This study aims to evaluate the effect of GCs on proliferation, differentiation, and metabolic function of brown adipocytes. Human brown adipocytes sourced from supraclavicular fat biopsies were grown in culture and differentiated to mature adipocytes. Human white adipocytes sourced from subcutaneous abdominal fat biopsies were cultured as controls. Effects of dexamethasone on growth, differentiation (UCP1, CIDEA, and PPARGC1A expression), and function (oxygen consumption rate (OCR)) of brown adipocytes were quantified. Dexamethasone $(1 \mu \mathrm{M})$ significantly stimulated the proliferation of brown preadipocytes and reduced that of white preadipocytes. During differentiation, dexamethasone (at $0.1,1$, and $10 \mu \mathrm{M}$ ) stimulated the expression of UCP1, CIDEA, and PPARGC1A in a concentration-dependent manner and enhanced by fourfold to sixfold the OCR of brown adipocytes. Isoprenaline $(100 \mathrm{nM})$ significantly increased $(P<0.05)$ expression of UCP1 and OCR of brown adipocytes. These effects were significantly reduced $(P<0.05)$ by dexamethasone. Thus, we show that dexamethasone stimulates the proliferation, differentiation, and function of human brown adipocytes but inhibits adrenergic stimulation of the functioning of brown adipocytes. We conclude that GCs exert complex effects on development and function of brown adipocytes. These findings provide strong evidence for an effect of GCs on the biology of human brown adipose tissue (BAT) and for the involvement of the BAT system in the metabolic manifestation of Cushing's syndrome.
\end{abstract}

\section{Key Words \\ - brown fat \\ - adipogenesis \\ - glucocorticoids \\ - thermogenesis}

\section{Introduction}

Brown adipose tissue (BAT) plays an important role in thermoregulation and energy balance in rodents. Thermogenesis in BAT is achieved by uncoupling protein 1 (UCP1), which dissociates ATP synthesis from the mitochondrial electrochemical gradient, dissipating energy in the form of heat. UCP1 is highly expressed in BAT in comparison to white adipose tissue (WAT). Mice lacking UCP1 develop obesity under thermoneutral conditions (Feldmann et al. 2009).

It has long been held that BAT in humans disappears rapidly after infancy. However, recent studies have provided unequivocal evidence that BAT in humans 
persists in adulthood and is present in most if not all adult humans (Lee et al. 2011). The metabolic significance of human BAT is under active investigation. The activity of BAT, as detected by metabolic imaging with fluorodeoxyglucose, is lower in obese subjects than in lean subjects. Virtanen et al. (2009) provided evidence that the activation of BAT in a healthy adult can account for significant energy utilization, equivalent to more than $4 \mathrm{~kg}$ of fat in a year. Recently, Yoneshiro et al. (2013) have observed that fat mass reduced significantly after 6 weeks of daily cooling for $2 \mathrm{~h}$ which significantly activated BAT. These observations provide persuasive evidence for a significant metabolic role of BAT in humans.

Glucocorticoids (GCs) play a critical role in the regulation of energy balance. Chronic GC excess, as exemplified in Cushing's syndrome, results in weight gain and obesity. GCs increase body fat mass through several mechanisms. They stimulate the recruitment of preadipocytes to mature white adipocytes (WAs), interacting with other hormones such as insulin to activate a program of WA differentiation. They act centrally in enhancing appetite by reducing leptin signaling (Madiehe et al. 2001).

The possibility that suppression of the functions of BAT mediates in part the weight gain induced by GCs has received little attention. In contrast to WAs, there is a paucity of information on the effects of GCs on BAT. It was reported nearly 20 years ago that GCs inhibited expression of UCP by BAT in rodents (Moriscot et al. 1993). Corticosterone inhibited both basal gene expression at thermoneutrality and the response to adrenergic stimulation and cold. Studies using rodent cell lines have observed that GCs downregulated UCP1 and the $\beta 3$ adrenergic receptor (Adrb3), which is characteristically highly expressed in BAT (Soumano et al. 2000).

The effect of GCs on BAT in humans is unknown. We have investigated the effects of GCs in a model of primary human adipocytes derived from biopsies of supraclavicular brown adipocyte (BA) depots (Lee et al. 2011). The brown preadipocytes proliferate and differentiate from stroma-vascular precursor stem cells over a period of 12 days into mature multilocular BAs expressing multilocular lipid droplets and classical BA transcripts.

\section{Materials and methods}

\section{Participants}

The biopsies of supraclavicular fat for studies of BA were obtained from 13 patients (two men and 11 women) who underwent neck surgery for thyroid nodules $(n=7)$, cancer $(n=3)$, hyperparathyroidism $(n=2)$, and Grave's disease $(n=1)$. These patients had a range of comorbidities and were treated with medications for depression, epilepsy osteoporosis, and diabetes. One patient was taking thyroxine and none were taking GCs. Abdominal subcutaneous fat for studies of WA was obtained from 11 patients (three men and eight women) for neoplasms $(n=5)$, hyperaldosteronism $(n=3)$, splenectomy $(n=1)$, cyst $(n=1)$, and fistula repair $(n=1)$. These patients had a range of comorbidities and were treated with medications for depression, epilepsy osteoporosis, and diabetes. Two patients were taking thyroxine and none were taking GCs.

\section{Primary preadipocyte culture}

Progenitor cells from the stromal vascular fraction (SVF) were isolated as described previously (Lee et al. 2011). Briefly, tissue was digested with collagenase (Life Technologies), filtered to remove debris, and incubated at $37{ }^{\circ} \mathrm{C}$ to allow mature adipocytes to settle in the upper phase. The SVF was collected from the lower phase, filtered, washed, and grown in $25 \mathrm{~cm}^{2}$ flasks until confluence was reached. Growth media consisted of DMEM with 10\% newborn calf serum (Life Technologies), $10 \mathrm{mM}$ HEPES, $1 \mathrm{nM}$ Tri-iodothyronine (Sigma), $100 \mathrm{nM}$ isoprenaline (Sigma), and $0.85 \mu \mathrm{M}$ insulin (Life Technologies). Upon reaching confluence, cells were differentiated in differentiation media (Lee et al. 2011). Mature adipocytes were characterized on the basis of lipid droplet accumulation and expression of relevant genes and proteins (see below).

\section{Preadipocyte proliferation}

Progenitor cells from the SVF were seeded at a density of 10000 cells/well (subconfluence) in 96-well plates in growth media. Cell density was measured using CellTiter 96 Non-Radioactive Cell Proliferation Assay (Promega) on day 1. To examine the effects of dexamethasone on proliferation, growth media were supplemented with dexamethasone ( 1 or $10 \mu \mathrm{M}$; Sigma) or left unsupplemented, and proliferation measured again on day 7 .

\section{Preadipocyte differentiation}

The effect of dexamethasone on differentiation of preadipocytes was examined by culturing confluent preadipocytes in differentiation media with and without dexamethasone for 12 days. Expression of genes and proteins, as a measure of BAT differentiation, were determined as described below.

Published by Bioscientifica Ltd 


\section{Quantitative real-time PCR}

RNA was isolated from undifferentiated (UD) cells and cells grown for $3,6,9$, and 12 days in differentiation media using a Purelink RNA Mini Kit (Life Technologies), and cDNA synthesized using a High Capacity cDNA RT Kit (Life Technologies). Fully validated TaqMan Gene Expression Assays (Life Technologies) were used with the SensiFAST Probe No-ROX Kit (Bioline, London, UK) and LightCycler 480 (Roche Applied Science) to determine relative gene expression by the comparative $C t$ method. Expression levels were determined relative to UD BAT, in order to determine the relative basal expression in WAT. Based on gene expression data, cells showed maximal response after 9 days of differentiation, and this time course was used for all subsequent experiments. UCP1, CIDEA, and PPARGC1A were examined as key molecular markers of BAT differentiation. We also investigated the effects of dexamethasone on the expression of HSD11B1 by BAs. As HSD11B1 controls local availability of GC by converting inactive cortisone to active cortisol, induction of HSD11B1 expression is likely to amplify the effects of GC itself.

\section{UCP1 immunohistochemistry}

Cells were fixed in 10\% formalin and immunohistochemistry was processed as described previously (Lee et al. 2011). Briefly, cells were blocked in 1\% BSA (Invitrogen) and incubated with a rabbit-anti-UCP1 antibody (Abcam, Cambridge, UK) or normal rabbit serum overnight. A HRP-conjugated secondary antibody was applied, followed by $3,3^{\prime}$-diaminobenzidine (Invitrogen).

\section{Adipocyte function}

This was assessed by quantifying oxidative respiratory rate in differentiation media. Preadipocytes isolated from brown fat biopsies were differentiated in $0,1.0$, or $10 \mu \mathrm{M}$ dexamethasone for 9 days in dedicated culture plates provided by the manufacturer that are required for undertaking measurement of oxygen consumption, as described below.

\section{Oxidative respiratory rate determination}

Oxygen consumption was determined using an XF24 Analyzer in conjunction with a statement Cell Mito Stress Test Kit (Seahorse Biosciences, North Billerica, MA, USA). Cell numbers were normalized after quantification using a CyQUANT NF Cell Proliferation Assay Kit (Invitrogen). Basal respiration was calculated by subtracting the oxygen consumption rate (OCR) of untreated cells from the OCR of cells after inhibition of the mitochondrial electron transport function with Antimycin A and Rotenone (inhibitors of complex III and I respectively). Uncoupled respiration was calculated by subtracting the OCR of cells treated with Oligomycin (an inhibitor of ATP synthase) from the OCR of cells after Antimycin A and Rotenone treatment. Maximum respiration was calculated by subtracting the OCR of cells treated with FCCP (carbonyl cyanide p-trifluoromethoxyphenylhydrazone, an electron transport chain uncoupling agent) from the OCR of cells treated with Antimycin A and Rotenone.

The basal OCR of the preadipocytes was determined after 9 days of differentiation. We also determined the effects of isoprenaline $(100 \mathrm{nM})$ on the OCR of these differentiated adipocytes in the presence and absence of dexamethasone. For these studies, day 9 differentiated adipocytes were cultured for $24 \mathrm{~h}$ in DMEM with $10 \%$ newborn calf serum (Life Technologies) and then treated with isoprenaline with or without dexamethasone $(1 \mu \mathrm{M})$ for $16 \mathrm{~h}$.

\section{Statistical analysis}

Statistical analysis was performed using GraphPad Prism (San Diego, CA, USA). Data are expressed as mean \pm s.E.m. Comparisons between BA and WA were performed using repeated-measure ANOVA with Dunnett's correction. All other comparisons were performed using nonparametric Kruskal-Wallis testing and Dunn's multiple comparison test. A $P$ value of $<0.05$ is considered statistically significant.

\section{Results}

\section{Effects of dexamethasone on brown preadipocyte proliferation}

To examine the effect of dexamethasone on proliferation, preadipocytes isolated from brown or white fat biopsies were cultured in the presence of 0,1 , or $10 \mu \mathrm{M}$ dexamethasone for 7 days. Treatment with $1 \mu \mathrm{M}$ dexamethasone significantly increased proliferation in brown preadipocytes and this effect approached statistical significance $(P=0.053)$ with $10 \mu \mathrm{M}$ (Fig. $1 \mathrm{~A})$. In contrast, dexamethasone significantly decreased proliferation in white preadipocytes at both 1 and $10 \mu \mathrm{M}$ (Fig. 1B).

Published by Bioscientifica Ltd 

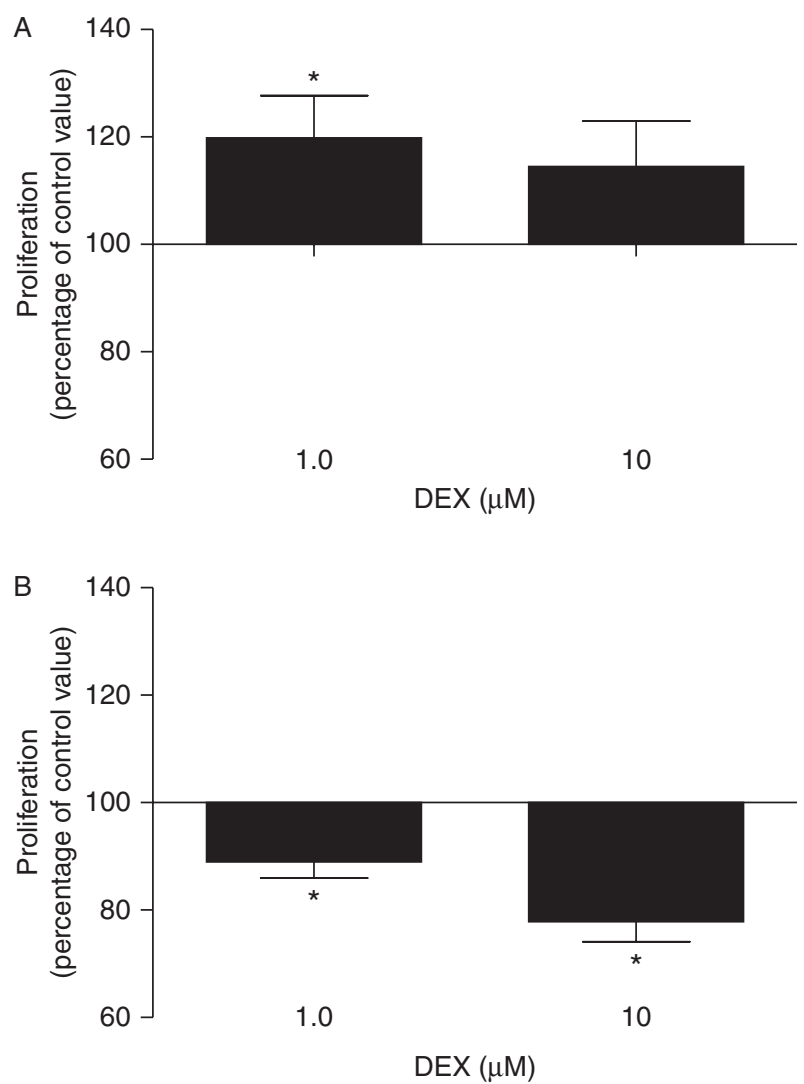

Figure 1

Effects of dexamethasone (DEX) on brown preadipocyte proliferation. Cell number of preadipocytes isolated from brown $(n=7)$ (A) or white $(n=9)$ (B) fat after 7 days of growth with 1.0 or $10 \mu \mathrm{M}$ dexamethasone. Data are expressed as the percentage of cells grown in the absence of dexamethasone. ${ }^{*} P<0.05$ versus control.

\section{Effect of dexamethasone on differentiation of brown preadipocytes}

Preadipocytes isolated from biopsies of either brown or white fat were cultured to confluence and placed in differentiation media for up to 12 days, and gene expression of UCP1, CIDEA, and PPARGC1A was quantified at days 3, 6, 9, and 12 (Fig. 2A, B and C). The changes were expressed in relation to that observed in UD preadipocytes immediately before placement in differentiation media. The time course and expression profile of brown preadipocyte differentiation were robust, consistently demonstrating maximal gene expression on day 9 . Subsequent experiments using dexamethasone at concentrations of $0,0.1,1.0$, or $10 \mu \mathrm{M}$ on cultured preadipocytes were therefore undertaken over 9 days. Dexamethasone treatment induced a dose-dependent stimulation on the expression of UCP1, CIDEA, and PPARGC1A in BA (Fig. 3A,
$B$ and $C$ ) inducing maximal changes of up to 336-fold for UCP1, 32-fold for CIDEA, and 2.8-fold for PPARGC1A. Expression of UCP1 protein in brown adipocytes differentiated in $0,1.0$, or $10 \mu \mathrm{M}$ dexamethasone was determined by immunohistochemistry. As observed for gene expression, expression of UCP1 protein was stimulated by dexamethasone in cells isolated from three individuals (Fig. 3D).

Low levels of UCP1 were expressed in WA and these did not change during differentiation. CIDEA, and PPARGC1A were more robustly expressed in WA, but once again did not change during differentiation (Fig. 2A, B and $\mathrm{C})$. Dexamethasone treatment stimulated expression of CIDEA but did not affect the expression of UCP1 or PPARGC1A (Fig. 3).

\section{Effect of dexamethasone on expression of HSD11B1}

Dexamethasone significantly increased the expression of HSD11B1 in both BA and WA; however, BA achieved significantly higher expression levels than WA (Fig. 4). Furthermore, overall levels of expression of HSD11B1 were markedly reduced in WA compared with BA (0.2-fold and 7 -fold respectively), relative to differentiated BAT in the absence of dexamethasone.

\section{Effect of dexamethasone on oxygen consumption}

Oxidative respiratory rate is an indicator of cellular metabolism, and expression of UCP1 in BAT underlies uncoupled respiration, giving rise to thermogenesis. Preadipocytes isolated from biopsies of brown fat were differentiated in $0,1.0$, or $10 \mu \mathrm{M}$ dexamethasone for 9 days in Seahorse XF24 culture plates. Figure 5A shows a representative OCR trace with a characteristic reduction in OCR upon the addition of Oligomycin (an inhibitor of ATP synthase; at time i), an increase in OCR with the addition of FCCP (an electron transport chain accelerator; at time ii), and decrease in OCR with Antimycin A and Rotenone (inhibitors of complex III and I respectively; at time iii). The addition of mitochondrial inhibitors provides an estimate of non-mitochondrial oxidation, which was subtracted from all other measurements to calculate the mitochondrial dependent respiration.

Uncoupled respiration is the hallmark of BAT function and is measured in the presence of Oligomycin which inhibits the activity of ATP synthase. Relative to cells differentiated in the absence of dexamethasone, both 1 and $10 \mu \mathrm{M}$ dexamethasone increased uncoupled OCR (Fig. 5B). Maximal respiratory rate, measured in the

Published by Bioscientifica Ltd 

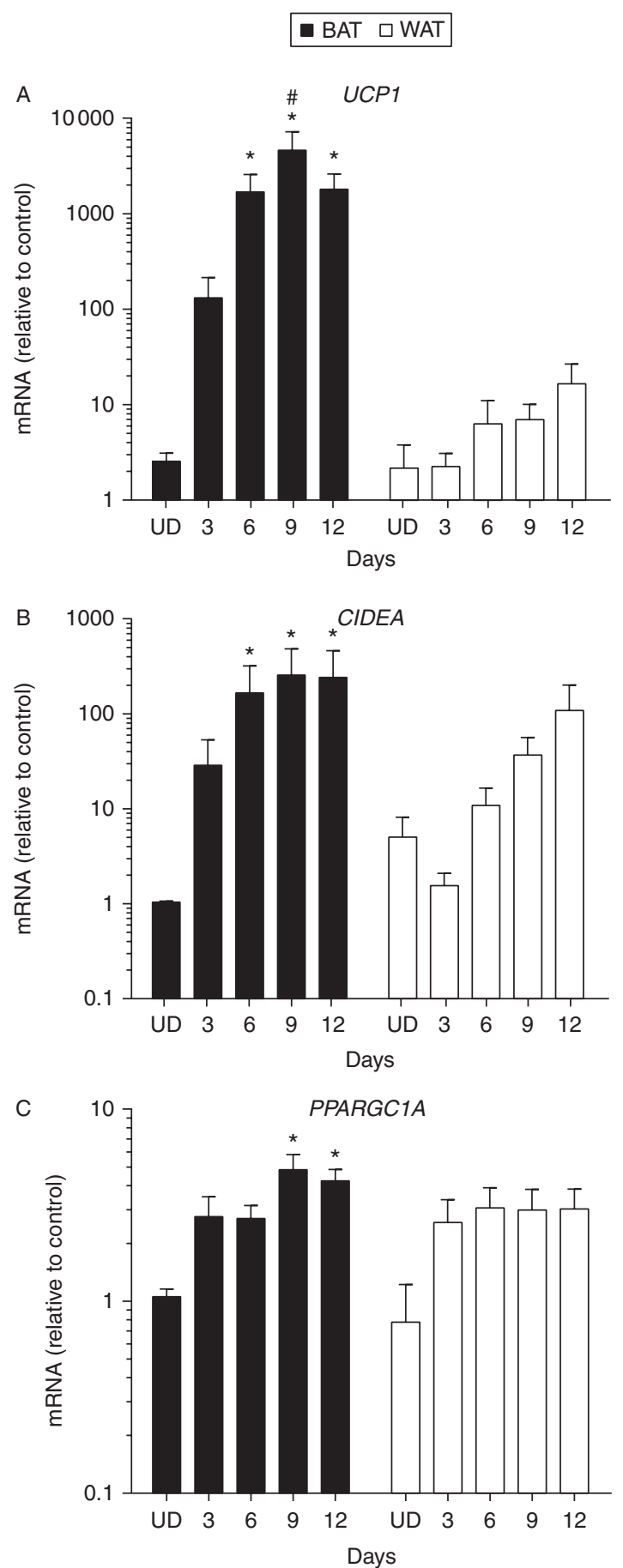

Figure 2

Abundance of mRNA of UCP1 ( $n=11$, BA and $n=5$, WA) (A), CIDEA $(n=10$, $\mathrm{BA}$ and $n=5, \mathrm{WA})$ (B), and PPARGC1A ( $n=10, \mathrm{BA}$ and $n=5$, WA) (C) during 12 days of differentiation. Gene expression in adipocytes isolated from brown (black bars) or white (white bars) fat prior to differentiation (undifferentiated (UD)) and after 3, 6, 9, and 12 days of differentiation. Data are expressed as fold changes relative to the values for UD brown adipocytes. *Denotes within-depot significance relative to control by oneway ANOVA: $P<0.05$ and ${ }^{\#}$ denotes between-depot significance by twoway ANOVA: $P<0.05$. presence of FCCP, is an indication of the capacity of the cells to respond to increased oxidative demand. $10 \mu \mathrm{M}$ dexamethasone significantly increased maximal respiratory rate (Fig. 5C).

\section{The effect of dexamethasone on adrenergic action}

BAT is stimulated by adrenergic activation via the sympathetic nervous system. To investigate the influence of dexamethasone on adrenergic regulation, brown preadipocytes were differentiated for 9 days and then incubated for $24 \mathrm{~h}$ in DMEM with NBCS. Cells were treated with $100 \mathrm{nM}$ isoprenaline, with or without $1 \mu \mathrm{M}$ dexamethasone, for $5 \mathrm{~h}$. Isoprenaline significantly stimulated the expression of UCP1, and this effect was abrogated by concurrent treatment with dexamethasone (Fig. 6A). Dexamethasone alone did not significantly affect the expression of $U C P 1$ over $5 \mathrm{~h}$.

BA function was examined by determining the OCR of cells stimulated with isoprenaline and/or dexamethasone for $16 \mathrm{~h}$. When expressed relative to the value for untreated cells, isoprenaline alone significantly increased uncoupled OCR (1.8-fold, $P=0.03)$, and $1 \mu \mathrm{M}$ dexamethasone significantly decreased isoprenaline-stimulated uncoupled mitochondrial oxidation and maximum oxidation (Fig. 6B and C).

\section{Discussion}

In this study of primary human BA obtained from supraclavicular fat biopsies, we demonstrate that dexamethasone stimulates proliferation of brown preadipocytes. It enhanced the expression of key BAT-related genes, namely UCP1, CIDEA, and PPARGC1A while also augmenting substantially the oxidative respiratory rate of brown preadipocytes during differentiation. However, dexamethasone significantly inhibited the oxidative rate of differentiated BAs stimulated by isoprenaline. In contrast, dexamethasone inhibited proliferation of white preadipocytes from subcutaneous abdominal fat. When cultured in brown-adipocyte-differentiation media, dexamethasone triggered a slight increase in the expression of CIDEA but not in the expression of UCP1 or PPARGC1A in white preadipocytes, the abundance of which was lower than that observed in brown preadipocytes undergoing differentiation. In summary, dexamethasone exerts divergent effects on proliferation of brown and white preadipocytes. It markedly stimulates the differentiation and basal oxidative rate of $\mathrm{BAs}$, but reduces adrenergic activation of BA function.

Published by Bioscientifica Ltd 


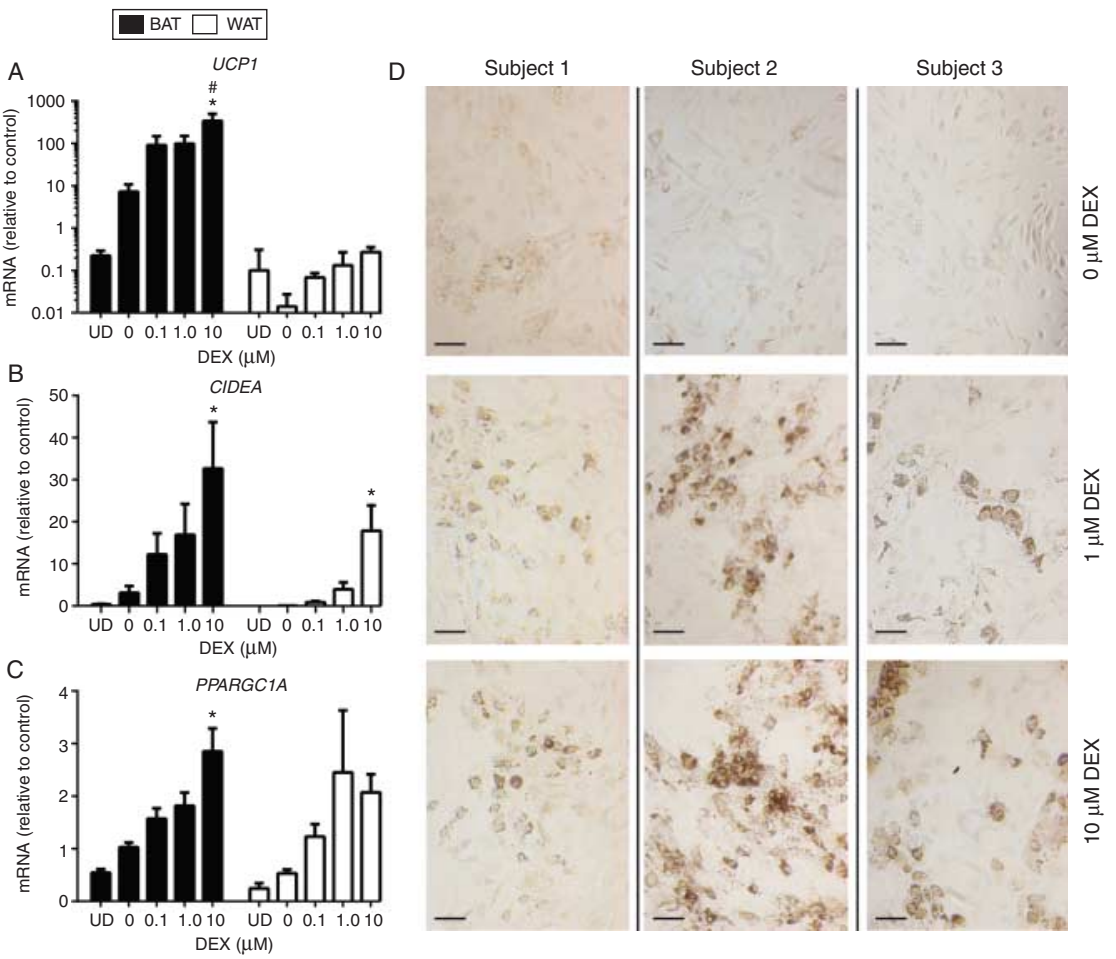

\section{Figure 3}

Effects of dexamethasone (DEX) on mRNA abundance of UCP1 $(n=9$, BA and $n=6$, WA) (A), CIDEA ( $n=6, \mathrm{BA}$ and $n=5$, WA) (B), and PPARGC1A ( $n=11, \mathrm{BA}$ and $n=3, \mathrm{WA}$ ) (C) in adipocytes isolated from brown (black bars) or white (white bars) fat prior to differentiation (undifferentiated (UD)) or adipocytes after 9 days of differentiation in the presence of $0,0.1$, 1.0 , or $10 \mu \mathrm{M}$ dexamethasone. Data are expressed as fold change relative to the values for UD brown adipocytes. *Denotes within-depot significance

Dexamethasone stimulated the proliferation of human brown preadipocytes in culture. To our knowledge, the effects of GCs on proliferation of brown preadipocytes from primary cultures or from human cell lines have not been reported, although quite extensive work with these cells has been published (Elabd et al. 2009, Nishio et al. 2012). Similarly, descriptions of the effects of GCs on rodent-derived BA cell lines have not been published (Ross et al. 1992). Contrary to the positive effect on proliferation of brown preadipocytes dexamethasone inhibited the expansion of white preadipocytes derived from subcutaneous adipose tissue. An inhibitory effect of GCs on proliferation of white preadipocytes has been observed in studies of rodents. Gregoire et al. (1991) reported that corticosterone and dexamethasone markedly reduced the growth of white adipocyte precursors derived from stroma-vascular cells from retroperitoneal fat of rats, an effect evident during the growth phase and after confluence. The authors of a recent review on the role of GCs in adipose tissue biology concluded that GCs exert Printed in Great Britain relative to the results for cells that had been treated with $0 \mu \mathrm{M}$ dexamethasone by one-way ANOVA: $P<0.05$ and ${ }^{\#}$ denotes between-depot significance by two-way ANOVA: $P<0.05$. UCP1 immunohistochemistry of brown preadipocytes isolated from three subjects and differentiated for 9 days in the presence of 0,1 , or $10 \mu \mathrm{M}$ dexamethasone (D). Magnification is $10 \times$. A full colour version of this figure is available at http://dx.doi.org/10. 1530/JOE-14-0538.

anti-proliferative effects on preadipocytes, but are required for full differentiation of adipocytes (Lee et al. 2014). Collectively, GCs appear to exert opposite effects on the proliferation of brown and white preadipocytes, stimulating growth of former and inhibiting that of the latter.

Dexamethasone stimulated the differentiation of human brown preadipocytes. It exerted a uniform effect on the expression of the BAT-marker genes UCP1, CIDEA, and PPARGC1A reaching significance at the maximal dose. This was accompanied by a marked increase in the levels of UCP1 protein and OCR and in particular uncoupled respiration, the hallmark of the thermogenic function of BAT. In white preadipocytes, dexamethasone did not stimulate expression of UCP1 or PPARGC1A expression, but increased the expression of CIDEA slightly. As CIDEA and PPARGC1A are also regulatory genes of white adipocytes, the effects of dexamethasone on these genes indicate a common effect on the differentiation of precursors of both brown and white adipocytes. There is strong evidence that GCs stimulate the differentiation 


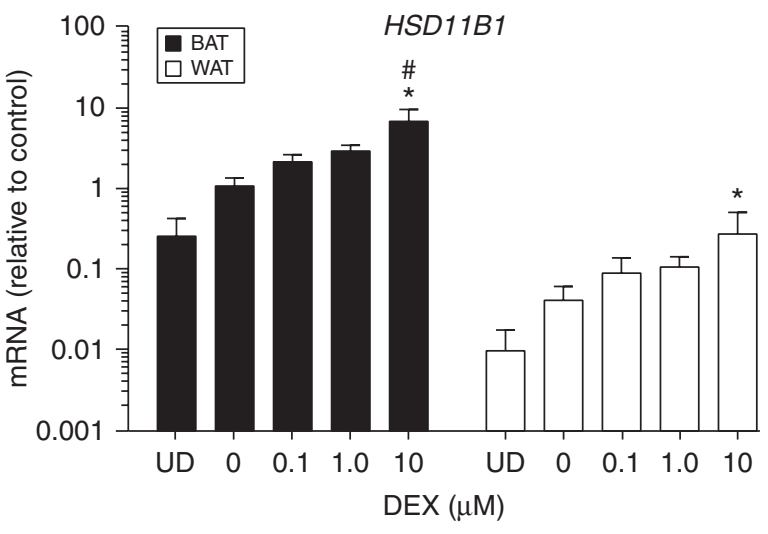

Figure 4

Effects of dexamethasone (DEX) on the abundance of mRNA HSD11B1. HSD11B1 mRNA in adipocytes isolated from brown (black bars) or white (white bars) fat prior to differentiation (undifferentiated (UD)) or after 9 days of differentiation in the presence of $0,0.1,1.0$, or $10 \mu \mathrm{M}$ dexamethasone, $n=4$. Data are expressed as fold change relative to UD brown adipocytes. *Denotes within-depot significance relative to cells not exposed to dexamethasone by one-way ANOVA: $P<0.05$ and "denotes between-depot significance by two-way ANOVA: $P<0.05$.

of white preadipocytes (Rebuffe-Scrive et al. 1988, AyalaSumuano et al. 2013, Lee et al. 2014). This effect is observed in primary cultures and cell lines of both humans and rodents (Lee et al. 2014). They act synergistically with insulin in simulating lipogenesis, triglyceride storage, and adipocyte hypertrophy, thus GCs appear to act concordantly in stimulating the differentiation of brown and white preadipocytes in contrast to their divergent effects on proliferation.

In our studies, treatment with dexamethasone induced expression of HSD11B1 in brown preadipocytes and white preadipocytes; however, this effect was significantly greater in brown preadipocytes. HSD11B1 regulates local concentrations of GCs by converting inactive cortisone to active cortisol. Unlike cortisol, dexamethasone, a synthetic GC agonist, does not rely on HSD11B1 for activation. Therefore, the differences in $H S D 11 B 1$ expression between brown and white adipocytes probably reflect differences in the effects of GCs between tissues rather than their local availability. A report by Lee et al. (2008) revealed that dexamethasone did not enhance the expression of HSD11B1 from white preadipocytes derived from subcutaneous abdominal fat. In contrast, HSD11B1 from omental fat was upregulated by dexamethasone, indicating the existence of depot-specific differences in responsiveness of HSD11B1 to GCs (Lee et al. 2008). Nevertheless, the observation that dexamethasone stimulates the expression of HSD11B1 is indicative of a novel stimulatory role for GCs in adipogenesis of BA, potentiated via a feed-forward pathway through the induction of HSD11B1.

The function of BAT in vivo is under the control of the sympathetic nervous system mediated by $\beta$ adrenergic activation. There is strong evidence that $\beta$ adrenergic

A
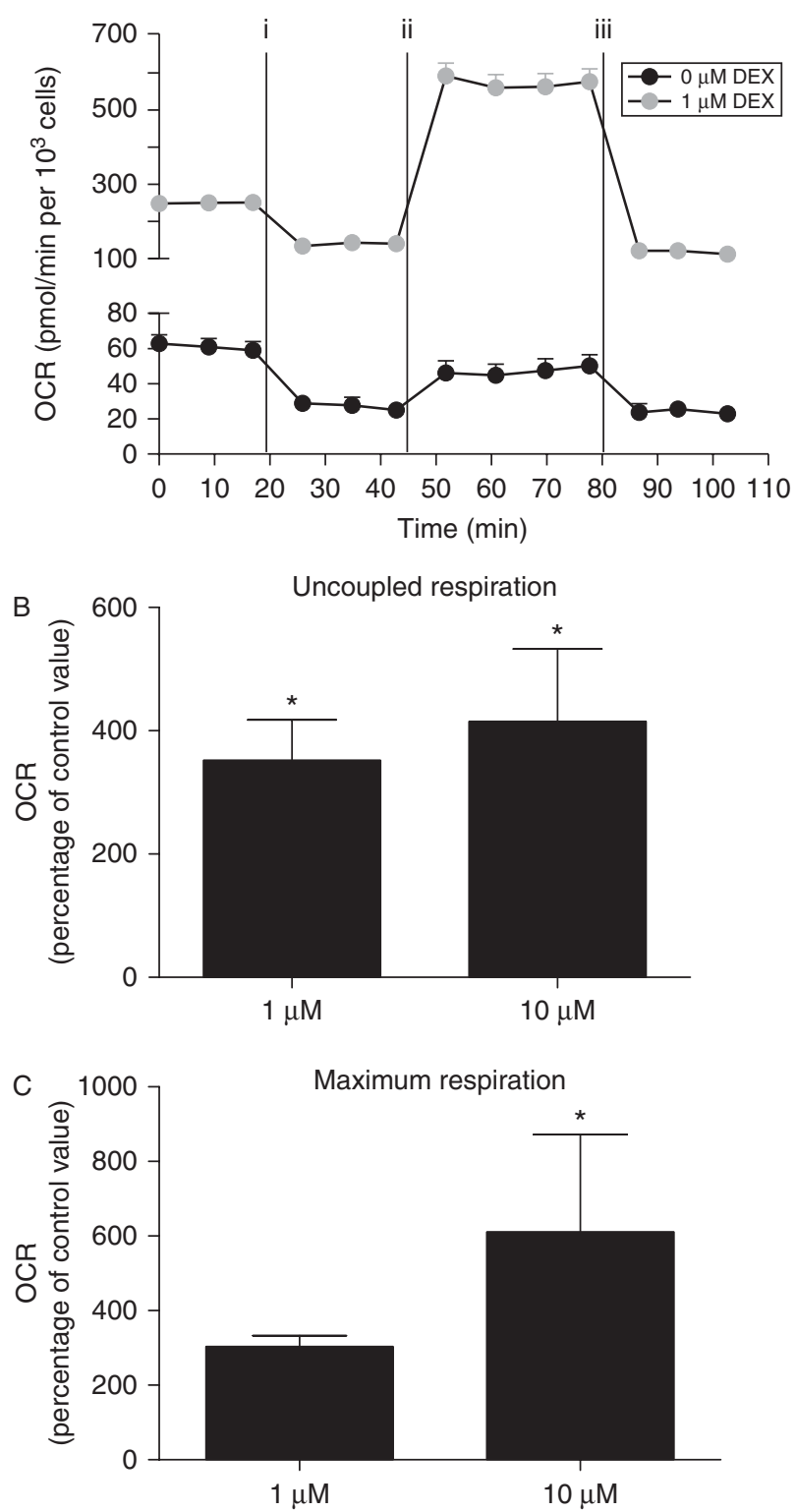

Figure 5

Effects of dexamethasone on oxygen consumption rate (OCR) in brown adipocytes. A representative (not normalized) OCR traces of cells differentiated in the presence of $0 \mu \mathrm{M}$ (black line) or $1 \mu \mathrm{M}$ (grey line) dexamethasone (A), demonstrating basal levels, the effect of Oligomycin (i), FCCP (ii), and Antimycin A and Rotenone (iii). Uncoupled OCR (B) and maximum OCR (C) of cells differentiated in the presence of 0,1 , or $10 \mu \mathrm{M}$ dexamethasone, $n=4$. Data are expressed as the percentage of OCR in cells differentiated in the absence of dexamethasone. ${ }^{*} P<0.05$ versus control.

Published by Bioscientifica Ltd 

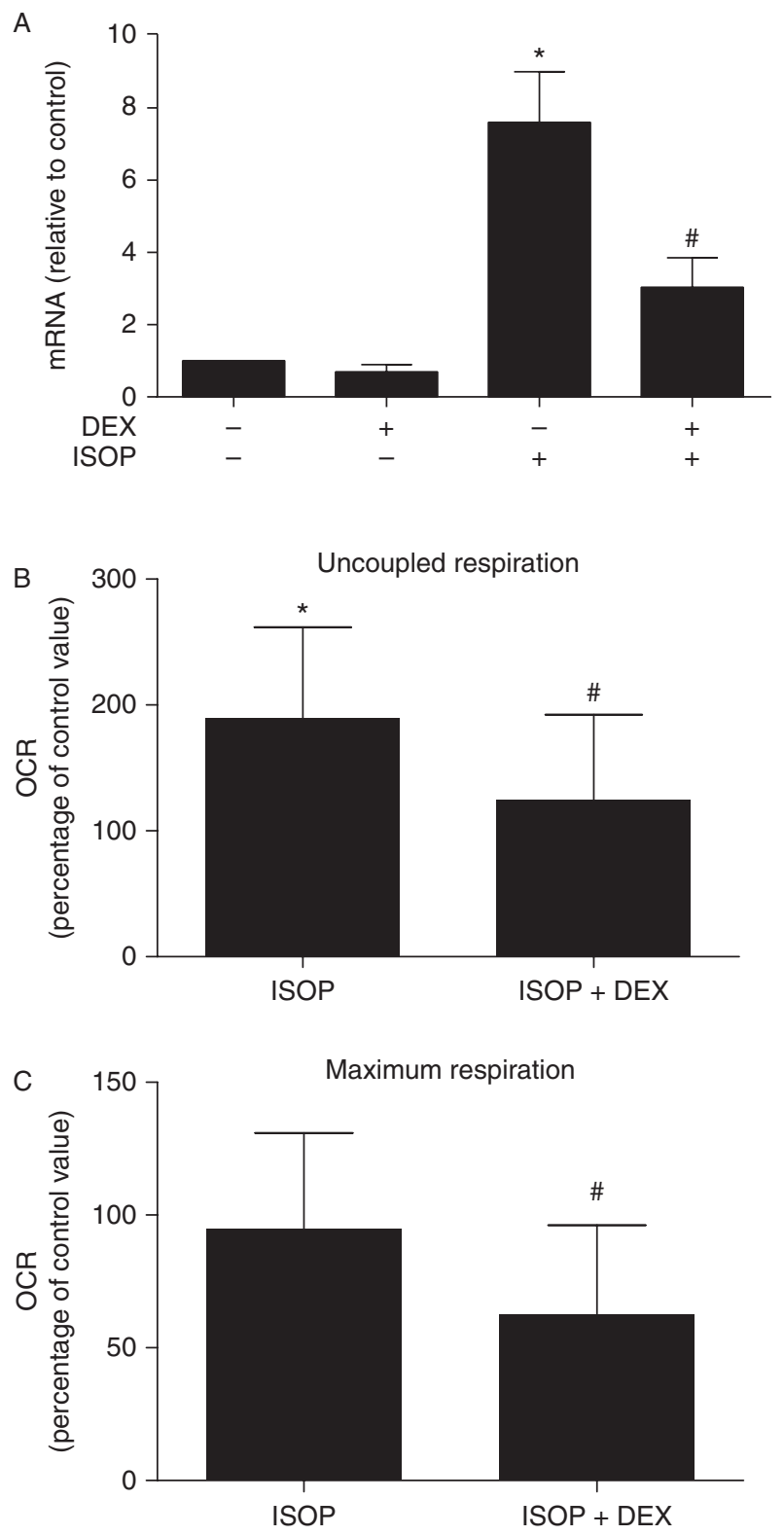

\section{Figure 6}

Separate and combined effects of isoprenaline (ISOP) and dexamethasone (DEX) on brown adipocytes. Expression of UCP1 in brown adipocytes differentiated for 9 days, and then stimulated with $100 \mathrm{nM}$ isoprenaline, with or without $1 \mu \mathrm{M}$ dexamethasone, for $5 \mathrm{~h}(\mathrm{~A}), n=4$. Uncoupled OCR (B) and maximum OCR (C) of cells stimulated with $100 \mathrm{nM}$ isoprenaline with or without $1 \mu \mathrm{M}$ dexamethasone, $n=5$. Data are expressed as the percentage of OCR in unstimulated cells. ${ }^{*} P<0.05$ versus control. ${ }^{\#} P<0.5$ ISOP versus ISOP+DEX.

system increases expression of UCP1 and stimulates the thermogenic function of BAT. Owing to the central stimulatory role of the adrenergic system on the function of BAT, we investigated the effect of dexamethasone on OCR of differentiated brown adipocytes stimulated by isoprenaline. Dexamethasone significantly reduced the isoprenaline-induced OCR of brown adipocytes, the major component of which was uncoupled oxidation. These observations on human brown adipocytes are supported by results from studies on rodents (Soumano et al. 2000, Rodríguez \& Palou 2004, Liu et al. 2013). The stimulation, by exposure to cold or isoprenaline, of expression of UCP1 of BAT from the intrascapular region is inhibited by GCs (Soumano et al. 2000). The combined results indicate that the stimulation of the function of BAT by the $\beta$ adrenergic activation is attenuated by GCs.

It is known that GC therapy causes weight gain, an effect often attributed to effects on appetite. We set out to determine whether weight gain might be attributed in part to inhibition of the abundance or function of BAT. We found, to our surprise, that dexamethasone enhanced the growth of brown preadipocytes, stimulating differentiation and basal metabolic activity, changes which would lead to negative rather positive energy balance. However, dexamethasone reduced the oxidative function of BAs under adrenergic stimulation, a situation that may mimic its effect in vivo. However, the present data do not allow us to deduce the net effect of GCs on the activity of BAT in vivo. It is also known that chronic GC excess causes an obese phenotype that characteristically includes fat hypertrophy around the base of the neck. These areas, including the supraclavicular fossa, correspond to the location of BAT depots in human adults. Based on our finding that dexamethasone stimulates the growth of brown adipocytes, it is conceivable that the hypertrophied neck fat observed in Cushing's syndrome repesents expanded depots of brown adipocytes. Our findings indicate the need for studies to investigate the wholebody in vivo effect of GCs on BAT as assessed by metabolic imaging with FDG, and the nature of neck fat in patients with GC excess.

In summary, the results of this first study, to our knowledge of the effects of GCs have demonstrated that dexamethasone stimulates the proliferation and the differentiation of human brown preadipocytes. Dexamethasone increased the oxidative function of these differentiated adipocytes by up to fourfold. However, dexamethasone inhibits the function of brown adipocytes under adrenergic stimulation. We conclude that the collective effects of GCs on BA biology are complex, punctuated by distinct stimulatory and inhibitory effects on the phases of development and regulated function. These novel findings indicate an important regulatory role of GCs in the biology of BAT and the probable involvement of the BAT system in the metabolic manifestation of Cushing's syndrome.

Published by Bioscientifica Ltd. 


\section{Declaration of interest}

The authors declare that there is no conflict of interest that could be perceived as prejudicing the impartiality of the research reported.

\section{Funding}

This study was supported by the National Health and Medical Research Council (NHMRC) of Australia.

\section{Author contribution statement}

All authors made contributions to the conception and design of the experiments. J L B and $\mathrm{H}$ A performed the research and developed the methods. J L B and KK Y H designed the research. All authors were involved in drafting and all approved the final version of the manuscript. $\mathrm{K} \mathrm{KY} \mathrm{H}$ is responsible for the integrity of the work as a whole.

\section{Acknowledgements}

The authors thank Mr A Mistry for technical assistance and Dr S Jalapu and $\mathrm{Mr} \mathrm{K}$ Carter for facilitating the collection of fat biopsies provided by Dr C Tan, Dr P Vuljovic, Dr D Wilkinson, Dr M Smithers, and Dr I Bennett during surgery. They thank DrJ Forbes for generously making the Seahorse XF24 Analyzer available for use in these studies.

\section{References}

Ayala-Sumuano J-T, Velez-delValle C, Beltran-Langarica A, MarschMoreno M, Hernandez-Mosqueira C \& Kuri-Harcuch W 2013 Glucocorticoid paradoxically recruits adipose progenitors and impairs lipid homeostasis and glucose transport in mature adipocytes. Scientific Reports 3 2573. (doi:10.1038/srep02573)

Elabd C, Chiellini C, Carmona M, Galitzky J, Cochet O, Petersen R, Pénicaud L, Kristiansen K, Bouloumié A, Casteilla L et al. 2009 Human multipotent adipose-derived stem cells differentiate into functional brown adipocytes. Stem Cells 27 2753-2760. (doi:10.1002/stem.200)

Feldmann HM, Golozoubova V, Cannon B \& Nedergaard J 2009 UCP1 ablation induces obesity and abolishes diet-induced thermogenesis in mice exempt from thermal stress by living at thermoneutrality. Cell Metabolism 9 203-209. (doi:10.1016/j.cmet.2008.12.014)

Gregoire F, Genart C, Hauser N \& Remacle C 1991 Glucocorticoids induce a drastic inhibition of proliferation and stimulate differentiation of adult rat fat cell precursors. Experimental Cell Research 196 270-278. (doi:10.1016/0014-4827(91)90261-R)

Lee M-J, Fried SK, Mundt SS, Wang Y, Sullivan S, Stefanni A, Daugherty BL \& Hermanowski-Vosatka A 2008 Depot-specific regulation of the conversion of cortisone to cortisol in human adipose tissue. Obesity $\mathbf{1 6}$ 1178-1185. (doi:10.1038/oby.2008.207)

Lee P, Swarbrick MM, Zhao JT \& Ho KK 2011 Inducible brown adipogenesis of supraclavicular fat in adult humans. Endocrinology 152 3597-3602. (doi:10.1210/en.2011-1349)

Lee M-J, Pramyothin P, Karastergiou K \& Fried SK 2014 Deconstructing the roles of glucocorticoids in adipose tissue biology and the development of central obesity. Biochimica et Biophysica Acta 1842 473-481. (doi:10.1016/j.bbadis.2013.05.029)

Liu J, Kong X, Wang L, Qi H, Di W, Zhang X, Wu L, Chen X, Yu J, Zha J et al. 2013 Essential roles of $11 \beta$-HSD1 in regulating brown adipocyte function. Journal of Molecular Endocrinology 50 103-113. (doi:10.1530/ JME-12-0099)

Madiehe AM, Lin L, White C, Braymer HD, Bray GA \& York DA 2001 Constitutive activation of STAT-3 and downregulation of SOCS-3 expression induced by adrenalectomy. American Journal of Physiology. Regulatory, Integrative and Comparative Physiology 281 R2048-R2058.

Moriscot A, Rabelo R \& Bianco AC 1993 Corticosterone inhibits uncoupling protein gene expression in brown adipose tissue. American Journal of Physiology 265 E81-E87.

Nishio M, Yoneshiro T, Nakahara M, Suzuki S, Saeki K, Hasegawa M, Kawai Y, Akutsu H, Umezawa A, Yasuda K et al. 2012 Production of functional classical brown adipocytes from human pluripotent stem cells using specific hemopoietin cocktail without gene transfer. Cell Metabolism 16 394-406. (doi:10.1016/j.cmet.2012.08.001)

Rebuffe-Scrive M, Krotkiewski M, Elfverson J \& Bjorntorp P 1988 Muscle and adipose tissue morphology and metabolism in Cushing's syndrome. Journal of Clinical Endocrinology and Metabolism 67 1122-1128. (doi:10.1210/jcem-67-6-1122)

Rodríguez A \& Palou A 2004 The steroid RU486 induces UCP1 expression in brown adipocytes. Pflügers Archiv: European Journal of Physiology 449 170-174. (doi:10.1007/s00424-004-1329-7)

Ross SR, Choy L, Graves RA, Fox N, Solevjeva V, Klaus S, Ricquier D \& Spiegelman BM 1992 Hibernoma formation in transgenic mice and isolation of a brown adipocyte cell line expressing the uncoupling protein gene. PNAS 89 7561-7565. (doi:10.1073/pnas.89.16.7561)

Soumano K, Desbiens S, Rabelo R, Bakopanos E, Camirand A \& Silva JE 2000 Glucocorticoids inhibit the transcriptional response of the uncoupling protein-1 gene to adrenergic stimulation in a brown adipose cell line. Molecular and Cellular Endocrinology 165 7-15. (doi:10.1016/S03037207(00)00276-8)

Virtanen KA, Lidell ME, Orava J, Heglind M, Westergren R, Niemi T, Taittonen M, Laine J, Savisto N-J, Enerbäck S et al. 2009 Functional brown adipose tissue in healthy adults. New England Journal of Medicine 360 1518-1525. (doi:10.1056/NEJMoa0808949)

Yoneshiro T, Aita S, Matsushita M, Kayahara T, Kameya T, Kawai Y, Iwanaga T \& Saito M 2013 Recruited brown adipose tissue as an antiobesity agent in humans. Journal of Clinical Investigation 123 3404-3408. (doi:10.1172/JCI67803)

Accepted 10 November 2014

Accepted Preprint published online 10 November 2014 http://joe.endocrinology-journals.org DOI: 10.1530/JOE-14-0538
() 2015 Society for Endocrinology Printed in Great Britain
Published by Bioscientifica Ltd 